\title{
Stimulation of Glucose Catabolism in Escherichia coli by a Potential Futile Cycle
}

\author{
RANJAN PATNAIK, ${ }^{1}$ WILLIAM D. ROOF, ${ }^{1,2}$ RYLAND F. YOUNG, ${ }^{2}$ AND JAMES C. LIAO ${ }^{1 *}$ \\ Department of Chemical Engineering ${ }^{1}$ and Department of Biochemistry and Biophysics, ${ }^{2}$ \\ Texas A\&M University, College Station, Texas 77843
}

Received 17 August 1992/Accepted 24 September 1992

\begin{abstract}
Fifteen-fold overexpression of phosphoenolpyruvate synthase (Pps) (EC 2.7.9.2) in Escherichia coli stimulated oxygen consumption in glucose minimal medium. A further increase in Pps overexpression to 30-fold stimulated glucose consumption by approximately 2 -fold and resulted in an increased excretion of pyruvate and acetate. Insertion of two codons at the PvuII site in the pps gene abolished the enzymatic activity and eliminated the above-described efiects. Both the active and the inactive proteins were detected at the predicted molecular weight by polyacrylamide gel electrophoresis. Therefore, the observed physiological changes were due to the activity of Pps. The higher specific rates of consumption of oxygen and glucose indicate a potential futile cycle between phosphoenolpyruvate (PEP) and pyruvate. A model for the stimulation of glucose uptake is presented; it involves an increased PEP/pyruvate ratio caused by the overexpressed Pps activity, leading to a stimulation of the PEP:sugar phosphotransferase system.
\end{abstract}

Phosphoenolpyruvate synthase (Pps) is a gluconeogenic enzyme that catalyzes the direct conversion of pyruvate to phosphoenolpyruvate (PEP), with the breakage of both of the phosphoanhydride bonds of ATP. This enzyme is required for growth on three-carbon substrates such as pyruvate and lactate $(6,7)$. The normal glycolytic flux from PEP to pyruvate in Escherichia coli is catalyzed by both pyruvate kinase and the PEP:sugar phosphotransferase system (PTS). A regulatory linkage between Pps and the PTS has been reported $(4,5,14)$. The fruR repressor, a negative regulator of the fru operon, which encodes the enzymes of the fructose PTS and fructose 1-phosphate kinase $(12,13,18)$, has also been shown to be required for pps transcription (14).

In principle, the coexistence of intracellular glycolytic and gluconeogenic activities could result in metabolic "futile cycling" (Fig. 1). Whether such cycling occurs and how it may be regulated or induced as a means of affecting metabolic flux are subjects of interest. With an unaltered genomic configuration, futile cycling may be difficult to detect. Little, if any, futile cycling between fructose 6-phosphate and fructose 1,6-diphosphate was detected in $E$. coli under either glycolytic (2) or gluconeogenic (10) conditions. However, the availability of multicopy clones of glycolytic or gluconeogenic genes may provide a way to induce futile cycling by titrating out the normal regulatory factors. Here we evaluate the consequences of expressing a multicopy clone of the pps gene in the presence of glucose as a carbon source. Since glucose uptake is mediated exclusively by the PTS, which is thought, in turn, to be regulated by the intracellular ratio of PEP to pyruvate (24), inappropriate Pps activity might be expected to have dramatic consequences for carbon flux. In this paper, these consequences are documented and then discussed in terms of the concept of futile cycling.

\section{MATERIALS AND METHODS}

Enzymes and chemicals. The following reagents were purchased from the indicated suppliers: D-glucose and all salts and metabolic enzymes, Sigma Chemical Co. (St. Louis,

\footnotetext{
* Corresponding author.
}

Mo.); protein assay reagents and chemicals for sodium dodecyl sulfate-polyacrylamide gel electrophoresis (SDSPAGE), Bio-Rad (Richmond, Calif.); isopropyl- $\beta-D-$ thiogalactopyranoside (IPTG), USB (Cleveland, Ohio); and restriction enzymes and T4 DNA ligase, Promega (Madison, Wis.).

E. coli strains and plasmids. The strains used are listed in Table 1. HG4 (pps pck) was a gift from A. H. Goldie, University of Saskatchewan, Saskatoon, Saskatchewan, Canada. Plasmid pUHE23-2, a pBR322 derivative, was obtained from H. Bujard, University of Heidelberg, Heidelberg, Germany. This plasmid is an IPTG-inducible expression control vector that contains a bacteriophage T7 early promoter (A1) modified to overlap two lac operators. This promoter is both tightly repressible and highly inducible by the addition of IPTG.

Media and growth conditions. Unless stated otherwise, the medium used in the strain construction was Luria broth (20), and the medium used in the physiological studies was M9 minimal medium (20) with $5 \mathrm{~g}$ of glucose per liter. Final concentrations of ampicillin and kanamycin were $50 \mu \mathrm{g} / \mathrm{ml}$. Samples were taken periodically from $E$. coli cultures grown at $37^{\circ} \mathrm{C}$ in a rotary shaker.

Cloning of the pps gene. The pps gene was cloned by use of kanamycin resistance $\left(\mathrm{Km}^{\mathrm{r}}\right)$ mini-Mu replicon $\mathrm{Mu}$ d5005 (16). A Mu lysate containing a DNA library of the pps ${ }^{+}$ strain HN198 was prepared with Mu d5005 as previously described (14). This lysate was used to transduce HG4 pps $p c k$ for growth in pyruvate minimal medium. The pps gene was present in mini-Mu plasmids isolated from $\mathrm{Pyr}^{+}$(growth on pyruvate) $\mathrm{Km}^{\mathrm{r}}$ colonies. pps was recovered on a $6.3-\mathrm{kbp}$ BamHI-HindIII fragment from one of these plasmids, pPS104, and subcloned into expression vector pUHE23-2 to yield pPS341 (Fig. 2a).

Codon insertion mutagenesis. The inactivation of the Pps enzyme was accomplished by use of a modified kanamycin resistance cassette from Chen et al. (3). The resistance cassette was obtained from pWJC3 by digestion with SmaI and was cloned into the PvuII site within the pps gene on pPS341 to yield pPS341K. For generation of a two-codon (GGGCCC) insertion in pps, pPS341K was digested with 


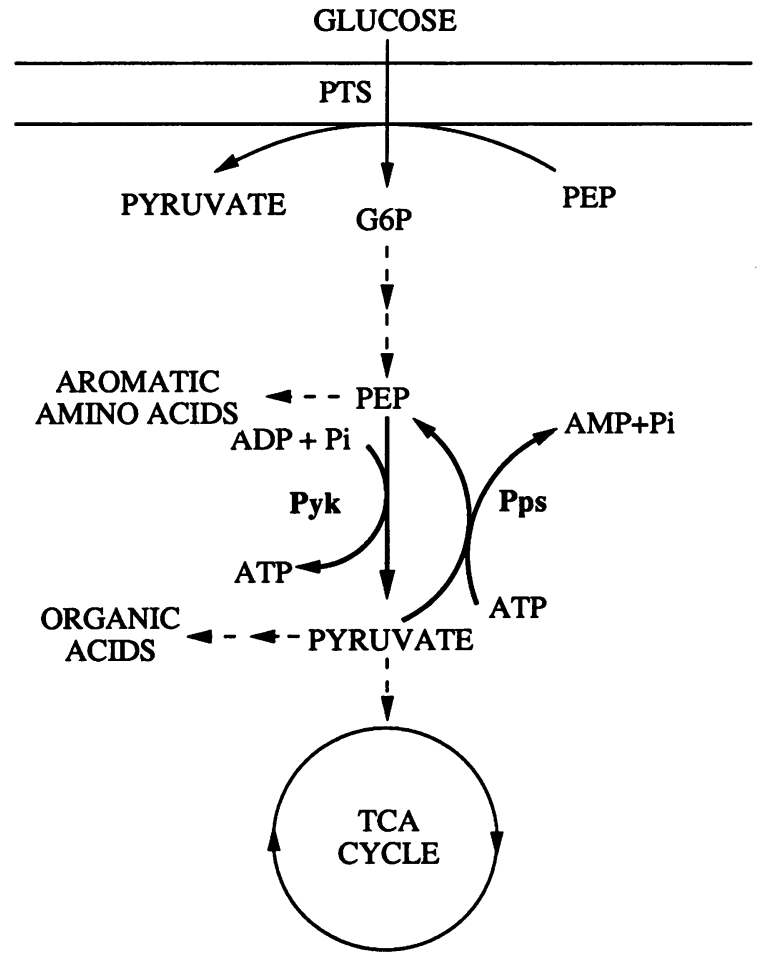

FIG. 1. Relative position of the PEP-pyruvate futile cycle in the overall scheme of metabolic pathways in $E$. coli. Broken lines indicate multiple enzymatic steps. Pyk, pyruvate kinase; G6P, glucose 6-phosphate; TCA, tricarboxylic acid.

ApaI and religated to form pPS341X1 (Fig. 2b). For generation of a 10-codon (ApaI-SalI-PstI-SalI-ApaI) insertion, pPS341K was digested with PstI and religated to form pPS341X4.

Preparation of cell extracts and enzyme assays. Cultures were harvested by centrifugation, resuspended in $5 \mathrm{mM}$ Tris-Cl-1 mM MgCl 2 , ( $\mathrm{pH} 7.4$ ), and ruptured by passage through a French pressure cell (SLM Aminco, Urbana, Ill.) at $16,000 \mathrm{lb} / \mathrm{in}^{2}$. Cell extracts were prepared as described previously (6). The Pps activity in these extracts was measured spectrophotometrically by monitoring the ATP-dependent disappearance of pyruvate (7). Total protein in the

TABLE 1. E. coli strains and plasmids used

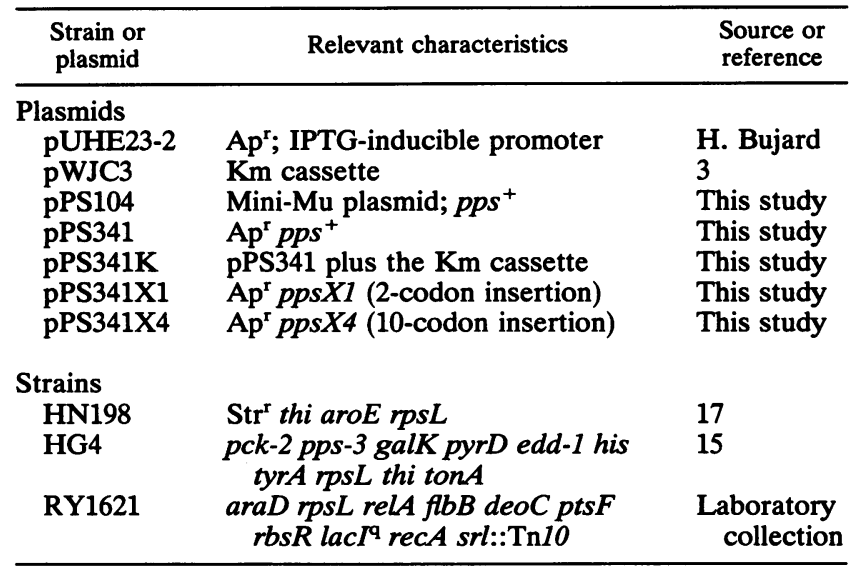

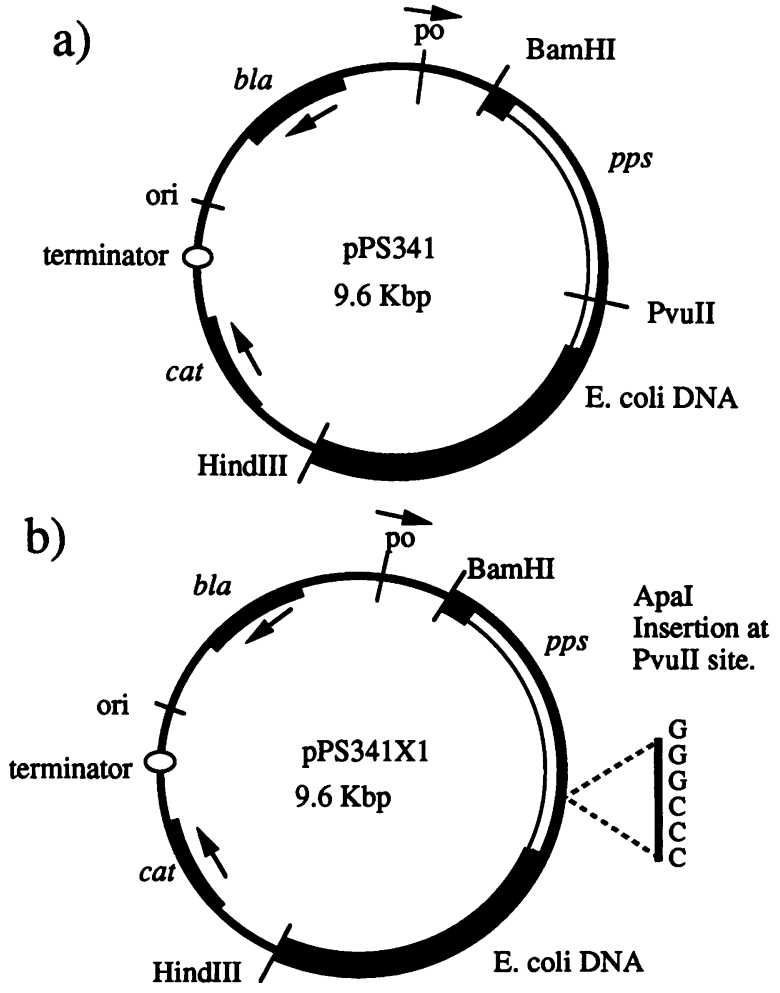

FIG. 2. Plasmid construction. (a) Plasmid pPS341. A 6.3-kbp fragment cut by BamHI and HindIII and containing the pps gene was subcloned into vector pUHE23-2 to obtain pPS341. Expression from the vector promoter is under the control of IPTG (see the text for details). The thin bar represents pUHE23-2 DNA, the thick filled bar represents the cloned fragment of $E$. coli DNA, and the thick open bar represents the probable position of the pps structural gene in the cloned fragment. (b) Plasmid pPS341X1. Inactivation of Pps was done by insertion of an ApaI site at the PvuII site in the pps gene. ori, origin of replication; po, promoter-operator.

extracts was determined with the Bio-Rad dye reagent (Bradford assay) and bovine serum albumin as the standard.

SDS-PAGE. An $8 \%$ polyacrylamide gel containing SDS was prepared, run, and Coomassie blue stained as described by Sambrook et al. (25). Whole-cell samples were prepared as described by Sambrook et al. (25) and normalized for protein concentrations before being loaded.

Fermentation product analysis. Pyruvate in the medium was determined colorimetrically essentially as described previously (11). The reaction of pyruvate with 2,4-dinitrophenyl hydrazine and the subsequent development of color after quenching with $\mathrm{NaOH}(10 \%$ [wt/vol]) are the bases of this assay. The color developed was measured by monitoring the $A_{445}$. Results were confirmed by an enzymatic assay with lactate dehydrogenase (9) and by high-pressure liquid chromatography (HPLC) analysis as described below. Ethanol produced was determined enzymatically on the basis of alcohol dehydrogenase-coupled NAD reduction under the conditions specified by the manufacturer of the reagents (Sigma).

Other products were analyzed by HPLC over an organic acid column (Aminex HPX-87H; Bio-Rad) with $0.01 \mathrm{~N}$ sulfuric acid as the eluant and at a flow rate of $0.6 \mathrm{ml} / \mathrm{min}$. The column temperature used was $60^{\circ} \mathrm{C}$, and the peaks were detected by measuring the UV $A_{210}$. 
TABLE 2. Specific activity of Pps in various constructs of strain RY1621

\begin{tabular}{lcc}
\hline \multicolumn{1}{c}{ Plasmid } & IPTG $^{b}$ & $\begin{array}{c}\text { Sp act }(\mu \mathrm{mol} / \mathrm{min} / \mathrm{mg} \\
\text { of protein })^{a} \pm 10 \%\end{array}$ \\
\hline None (host) & - & 0.02 \\
pUHE23-2 & - & 0.02 \\
pPS341 & - & 0.29 \\
pPS341 & + & 0.60 \\
pPS341X1 & - & 0.03 \\
pPS341X1 & + & 0.03 \\
pPS341X4 & - & 0.02 \\
pPS341X4 & + & 0.03 \\
\hline \multicolumn{2}{c}{${ }^{a}$ Specific activity was measured at $25^{\circ} \mathrm{C} 5$ h after inoculation $\left(\mathrm{OD}_{550}, 1\right.$ to } \\
2). ${ }_{b}$ IPTG (1 mM) was added $1.5 \mathrm{~h}$ after inoculation $\left(\mathrm{OD}_{550}, 0.08\right.$ to 0.1$)$.
\end{tabular}

Measurement of the glucose consumption rate. The glucose concentration in the culture supernatant was determined by the dinitrosalicylic acid assay for total reducing sugars (19). The results were confirmed by an NADPH-coupled enzyme assay with hexokinase and glucose 6-phosphate dehydrogenase (1). A polynomial curve was fitted to each set of data. The slope of the curve, which represented the glucose disappearance rate at each time point was evaluated. The specific glucose consumption rate was calculated as the rate of glucose disappearance in the medium divided by the cell density (optical density at $550 \mathrm{~nm}\left[\mathrm{OD}_{550}\right]=0.36 \mathrm{mg}$ of dry cells per ml) measured at the corresponding time.

Measurement of the oxygen consumption rate. Oxygen consumption curves were obtained by use of a YSI model 5300 biological oxygen monitor supplied by Yellow Springs Instruments Inc. (Yellow Springs, Ohio). Five milliliters of a culture with a known $\mathrm{OD}_{550}$ was transferred to an airtight sample chamber, in which a Clark-type polarographic oxygen probe monitored the disappearance of oxygen from the medium as a function of time. The linearity between the oxygen disappearance rate and the cell density at different dilutions was demonstrated to verify the results. The initial rate of oxygen disappearance, which remained constant for at least $2 \mathrm{~min}$, was divided by the cell density to obtain the specific oxygen consumption rate. The oxygen solubility in the medium at $37^{\circ} \mathrm{C}$ was taken to be $0.264 \mathrm{mM}$ in the calculation.

\section{RESULTS}

Expression of the pps gene. Growth of a culture of $\operatorname{lacl}^{\mathrm{q}}$ strain RY1621 carrying pPS341 $\left(\right.$ pps $\left.^{+}\right)$resulted in a 15-fold increase in Pps activity over that in the same host carrying the vector plasmid (Table 2). The addition of the inducer IPTG resulted in a further twofold induction. Presumably, the high level of expression in the absence of an inducer reflects the transcription of pps from a linked promoter in the 6.3-kbp cloned fragment.

Inactivation of the pps gene. To control for the effects of the multicopy plasmid and the metabolic load caused by protein overproduction, we inactivated the Pps enzyme by inserting additional codons into the $P v u I I$ site in the structural gene. The PvuII cleavage site is between codons 708 and 709 , according to D. L. Holzschu and coworkers (GenBank accession number M69116) or between codons 707 and 708, according to Niersbach et al. (23). Therefore, the GGGCCC insertion would code for a glycine-proline insertion into the Pps protein. The Pps activities of RY1621/pPS341X1 (2codon insertion in pps) and RY1621/pPS341X4 (10-codon

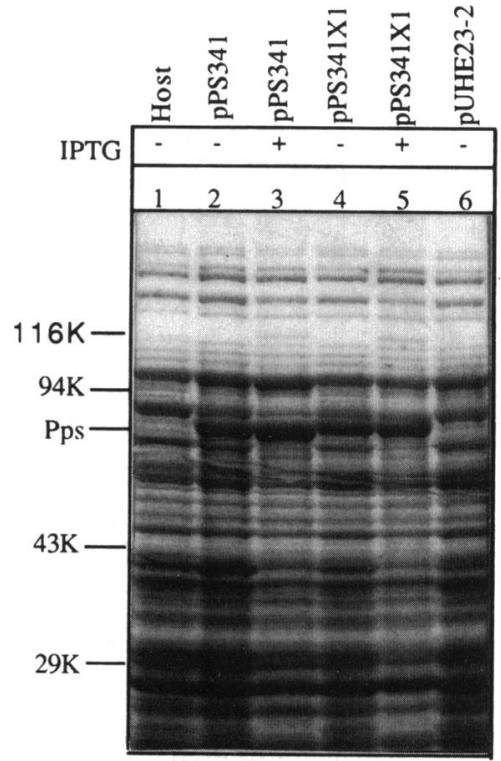

FIG. 3. SDS-PAGE. The overproduction of Pps, both in active and inactive forms in the presence of glucose, is shown. Plasmids pUHE23-2, pPS341, and pPS341X1 were transformed into the same host, RY1621 (Table 1). + and -, with and without induction by 1 mM IPTG, respectively.

insertion in pps) were shown to be at the background level (Table 2), either with or without induction.

Identification of the products of pps wild-type and mutant alleles. The Pps protein was identified as a ca. 80,000- $M_{\mathrm{r}}$ species by SDS-PAGE (Fig. 3), in good agreement with the $77,000-M_{\mathrm{r}}(22)$ and $84,000-M_{\mathrm{r}}(14)$ estimates reported previously and with the size of the pps reading frame ( 792 codons) (23). The amount of this species, as assessed by SDS-PAGE band intensity, increased about twofold upon induction, in good agreement with the induction of Pps activity (Fig. 3, lanes 2 and 3 ). A similar twofold increase was observed for the inactive product of the ppsX1 allele (2-amino-acid insertion) (Fig. 3, lanes 4 and 5). No band could be identified as the product of the pps $X 4$ allele, indicating that the insertion of 10 amino acids conferred proteolytic instability on the Pps protein.

Pps overexpression causes increased fermentation product excretion. Growth of the Pps-overproducing strain (RY1621/ pPS341) was retarded after the addition of IPTG (Fig. 4). This phenomenon was not due to Pps activity, since the inactivated clone (pPS341X1) also showed growth retardation. HPLC and an enzymatic assay of the supernatants from Pps-overproducing cultures revealed that a number of fermentation products, including pyruvate, acetate, lactate, formate, succinate, and ethanol, were present. Remarkably, induction of the multicopy pps $^{+}$clone resulted in a $>10$-fold increase in the level of excreted pyruvate over the uninduced level (data not shown). The extracellular concentration of pyruvate was about $23 \mathrm{mM}$, equivalent to $41 \%$ of the carbon in glucose being excreted as pyruvate.

To assess the Pps-induced physiological changes at the quasi-steady state, we took aliquots of the cultures shown in Fig. 4 at $10 \mathrm{~h}$ and diluted them in glucose minimal medium with $1 \mathrm{mM}$ IPTG to an $\mathrm{OD}_{550}$ of 0.3 . The growth rates of these cultures remained constant for at least $2 \mathrm{~h}$ (Fig. 5a), and the product formation patterns (Fig. 5b and c) showed induced pyruvate and acetate excretion by overexpressed 


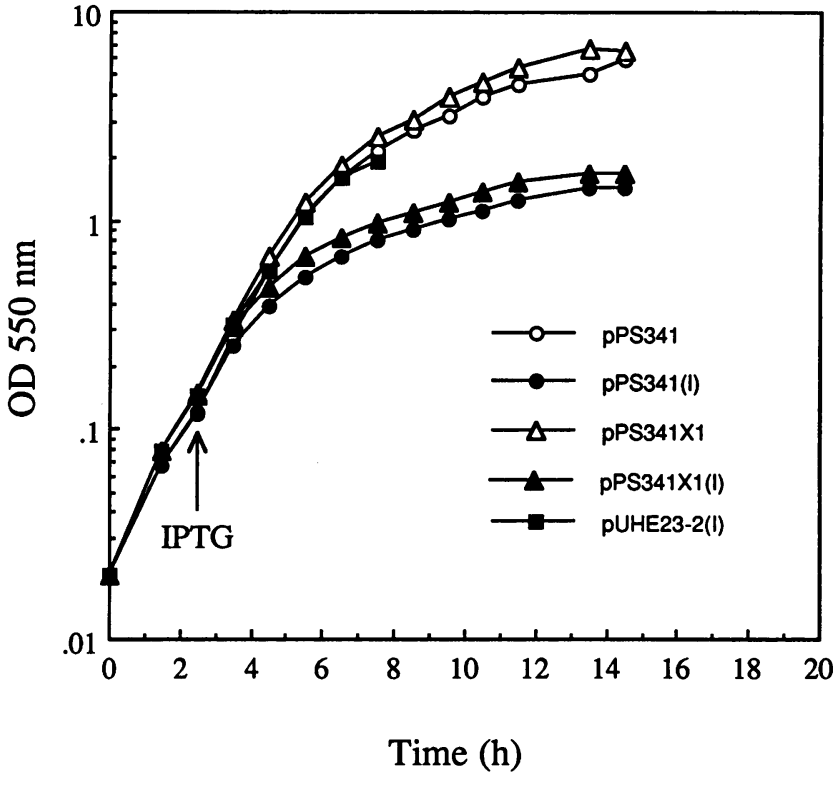

FIG. 4. Growth curves in glucose minimal medium. Strain RY1621 contained the plasmids shown. I, induction by IPTG (1 $\mathrm{mM})$.

Pps. The levels of lactate, ethanol, formate, and succinate produced during quasi-steady-state growth (data not shown) were insignificant compared with those of the major products. The reciprocal yield coefficients (stoichiometry) and the specific product formation rates are tabulated in Tables 3 and 4 , respectively.

Overexpressed Pps stimulates glucose and oxygen consumption. The inducible increase in fermentation product excretion with the $\mathrm{pps}^{+}$clone correlated with an increase in specific glucose uptake (Fig. 6). The induced culture with multicopy $\mathrm{pps}^{+}$(RY1621/pPS341) showed a ca. two- to threefold increase in specific glucose uptake over control uptake.

The oxygen consumption rate was monitored as described in Materials and Methods. The oxygen concentrations in the assay chamber were measured as a function of time, and the specific oxygen consumption rates were calculated (Table 4). The strain with multicopy $\mathrm{pps}^{+}$(RY1621/pPS341), under both induced and uninduced conditions, showed a twofold increase in the specific oxygen consumption rate over the isogenic strain carrying the vector plasmid (RY1621/ pUHE23-2) and a ca. fourfold increase in this rate over the induced strain with multicopy ppsX1 (RY1621/pPS341X1). The increase in oxygen consumption and the decrease in glucose yield suggest an increased ATP turnover caused by Pps activity.

\section{DISCUSSION}

In conjunction with pyruvate kinase, Pps catalyzes a potential futile cycle between PEP and pyruvate. In addition, the simultaneous function of the PTS and Pps results in a net reaction of sugar transport and phosphorylation at the expense of two phosphate bonds in ATP (sugar + ATP $\rightarrow$ sugar-P $\left.+A M P+P_{i}\right)$, a reaction that is in effect equivalent to the coupling of a futile cycle to sugar transport and phosphorylation. It is thus expected that the expression of Pps must be highly regulated. However, even in the pres-
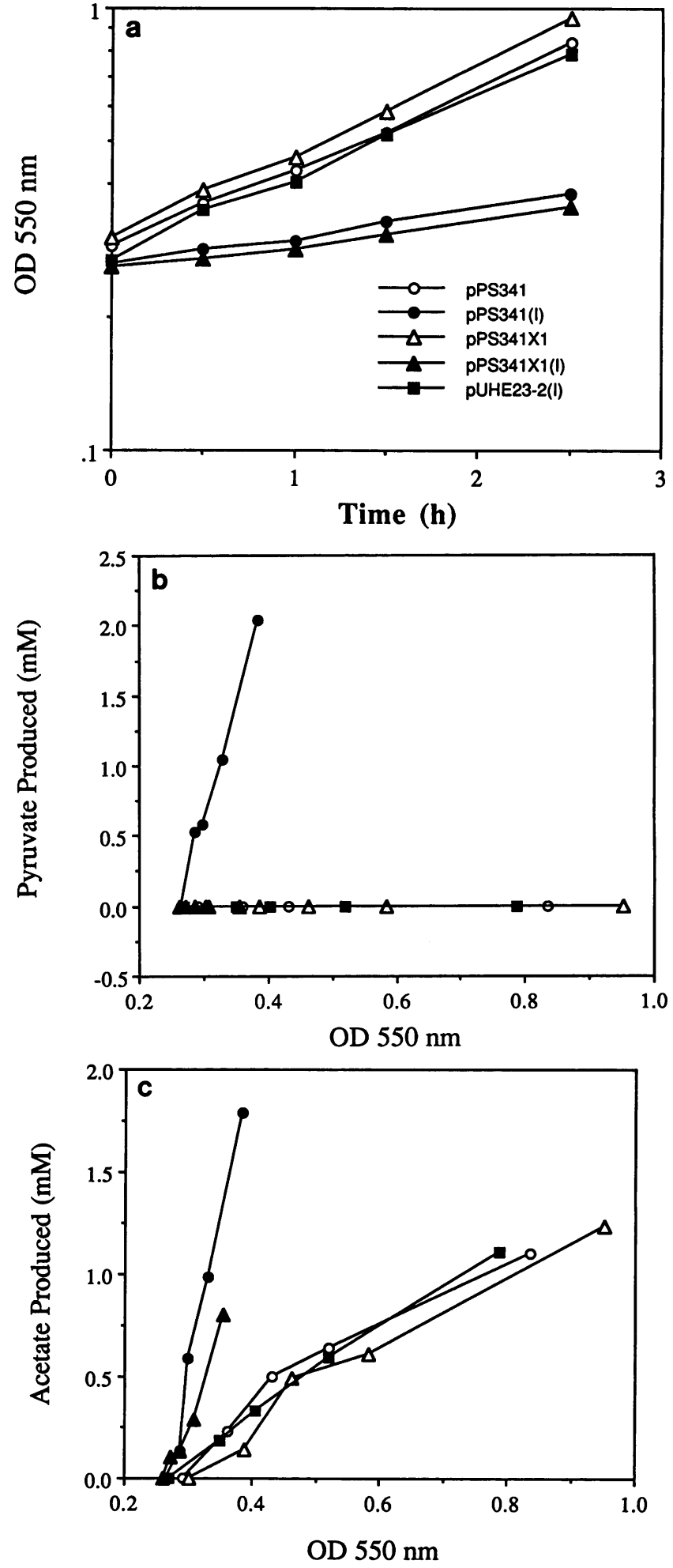

FIG. 5. Growth curves and product formation of the diluted cultures. (a) Strain RY1621 contained the plasmids shown. Cultures were initiated by diluting aliquots of the 10-h cultures shown in Fig. 4 in glucose minimal medium (with $1 \mathrm{mM}$ IPTG, as indicated by I). (b and c) The metabolites produced were calculated as the concentrations at each time point minus the initial concentrations carried over from the prediluted cultures. The standard deviation of each product measurement was about $10 \%$ of the measured value. 
TABLE 3. Reciprocal yields of metabolites ${ }^{a}$

\begin{tabular}{lcrrcccc}
\hline & \multicolumn{7}{c}{ Reciprocal yield of: } \\
\cline { 2 - 8 } Plasmid $^{b}$ & Glucose & $\begin{array}{c}\text { Pyru- } \\
\text { vate }\end{array}$ & Acetate & Formate & Lactate & Ethanol & $\begin{array}{c}\text { Succi- } \\
\text { nate }\end{array}$ \\
\hline pPS341 & 17 & 0 & 5.6 & 0 & 0 & 0.85 & 0 \\
pPS341 (I) & 87 & 50 & 42 & 5 & 0 & 4.7 & 0 \\
pPS341X1 & 16 & 0 & 5.6 & 0 & 0 & 0.34 & 0 \\
pPS341X1 (I) & 33 & 0 & 27 & 0 & 0 & 5.1 & 0 \\
pUHE232 (I) & 14 & 0 & 5.6 & 0 & 0 & 0.43 & 0 \\
\hline
\end{tabular}

a Yields were calculated as the change in the cell mass divided by the change in the level of the metabolite (grams [dry weight] of cells/millimoles of metabolite). The host strain was RY1621.

$b$, induction by IPTG $(1 \mathrm{mM})$.

ence of fructose, basal Pps activity is about $40 \%$ the activity in the presence of lactate (14). This phenomenon suggests that the cell may have a mechanism for tolerating or avoiding futile cycling.

The observed physiological changes caused by Pps overproduction can be summarized in the following two-stage model. (i) A moderate increase in Pps activity (below 0.3 $\mu \mathrm{mol} / \mathrm{min} / \mathrm{mg}$ ) in the presence of glucose stimulates oxygen consumption. At this stage, the increase in oxygen consumption is sufficient to compensate for the potential futile cycling, presumably by increasing the synthesis of ATP via oxidative phosphorylation. No increase in glucose uptake is necessary until the respiration capacity is saturated. Therefore, the uninduced culture of the multicopy $\mathrm{pps}^{+}$strain (RY1624/pPS341) shows a significant increase in oxygen consumption but no significant increase in glucose consumption or product formation. (ii) A further increase in Pps activity stimulates glucose uptake and causes an increase in the formation of fermentation products. At this stage, the respiration pathways are already saturated and the increase in glycolytic flux must result in the excretion of fermentation end products, such as pyruvate and acetate.

The stimulation of oxygen consumption has also been observed for $E$. coli cultures grown in glucose with the addition of carbonyl cyanide $m$-chlorophenylhydrazone (21), which creates proton leakage. In both cases, the cell responds to energy stress by increasing the respiration rate. However, the detailed molecular mechanism of Pps-induced oxygen consumption is unknown.

The increase in glucose uptake with high Pps activity may be achieved by an increase in the PEP/pyruvate ratio, as a result of Pps mass action, with or without active futile

TABLE 4. Specific consumption or production rates ${ }^{a}$

\begin{tabular}{|c|c|c|c|c|c|c|}
\hline \multirow{2}{*}{ Plasmid $^{b}$} & \multicolumn{6}{|c|}{ Consumption or production of: } \\
\hline & Oxygen $^{c}$ & Glucose $^{d}$ & $\begin{array}{l}\text { Pyru- } \\
\text { vate }\end{array}$ & Acetate & Formate & Ethanol \\
\hline $\begin{array}{l}\text { pPS341 } \\
\text { pPS341 (I) } \\
\text { pPS341X1 } \\
\text { pPS341X1 (I) } \\
\text { pUHE232 (I) }\end{array}$ & $\begin{array}{c}11,14 \\
10,16 \\
5,7.5 \\
2.5,3.5 \\
4.5,9.5\end{array}$ & $\begin{array}{l}7 \\
13 \\
6.5 \\
5 \\
6\end{array}$ & $\begin{array}{l}0 \\
7.5 \\
0 \\
0 \\
0\end{array}$ & $\begin{array}{l}2.4 \\
6.3 \\
2.4 \\
4.0 \\
2.4\end{array}$ & $\begin{array}{l}0 \\
0.8 \\
0 \\
0 \\
0\end{array}$ & $\begin{array}{l}0.36 \\
0.70 \\
0.14 \\
0.76 \\
0.18\end{array}$ \\
\hline
\end{tabular}

a Calculated as [d(metabolite) $/ d t] /$ cell mass $=\mu /$ metabolite yield (millimoles of metabolite produced per hour per gram of dry cell weight), where $\mu$ is the specific growth rate (1/hour). The host strain was RY1621.

$b$ I, Induction by IPTG $(1 \mathrm{mM})$.

$c$ Measured at 0.5 and $2 \mathrm{~h}$, respectively.

${ }^{d}$ Measured at $1.5 \mathrm{~h}$.

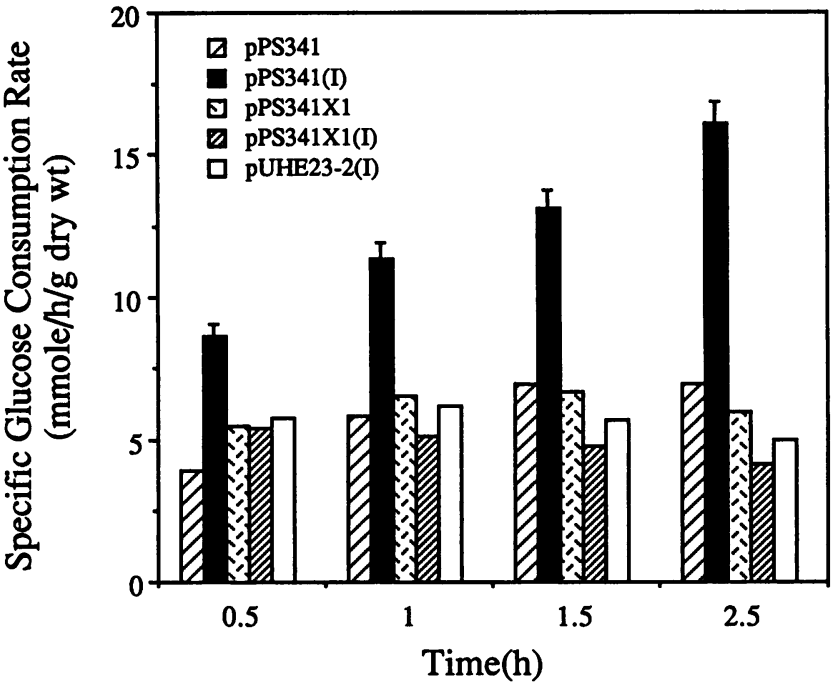

FIG. 6. Specific glucose consumption rate calculated from the slope of the residual glucose curve and the cell density measured at the corresponding time. I, addition of $1 \mathrm{mM}$ IPTG.

cycling, as follows. The increased ratio increases the phosphorylated forms of enzyme I, HPr, and enzyme III ${ }^{\text {Glc }}$ of the PTS. These increases in the phosphorylated PTS enzymes cause an increase in glucose uptake. It has been suggested that the PEP/pyruvate ratio could serve as a rate-controlling parameter in the PTS (24).

The increase in pyruvate and acetate excretion may be attributed to the high glycolytic flux, which saturates the tricarboxylic acid cycle and "overflows" to these products. Pyruvate is produced as the major product instead of lactate or ethanol. The net NADH gain (2 NADH/glucose) in converting glucose to pyruvate can be oxidized through the cytochrome system and can generate more ATP to compensate for the potential futile cycling. The possibility of Pps acting in the reverse direction (from PEP to pyruvate) is unlikely, considering the high pyruvate concentration in the medium and the low activity in the reverse direction $(<30 \%$ that in the forward direction), even at the optimal $\mathrm{pH}(6.8)$ for pyruvate formation (8). In addition to metabolic overflow, the induced acetate production is also consistent with the futile cycling hypothesis, since the conversion of pyruvate to acetate is accompanied by ATP production.

To explain the observed effects in detailed mechanisms, investigators must measure key intracellular metabolites. It will also be extremely useful to demonstrate the existence of active futile cycling between PEP and pyruvate and the mechanisms for regulating it. Experiments along these lines are currently under way in our laboratory.

\section{ACKNOWLEDGMENTS}

We thank Mary E. Clark for technical assistance.

This material is based in part on work supported by National Science Foundation grant BCS-9009851 (to J.C.L.) and by the Texas Advanced Technology Program under grant 999903156 (to J.C.L. and R.F.Y.).

\section{REFERENCES}

1. Bergmeyer, H. P., E. Bernt, F. Schmidt, and H. Strok. 1974. D-Glucose: determination with hexokinase and glucose-6-phosphate dehydrogenase, p. 1196-1201. In H. U. Bergmeyer and K. Gawehn (ed.), Methods of enzymatic analysis, vol. 3. Academic Press, Inc., New York. 
2. Chambost, J. P., and D. G. Fraenkel. 1980. The use of $6-{ }^{14} \mathrm{C}$ labeled glucose to assess futile cycling in Escherichia coli. J. Biol. Chem. 255:2867-2869.

3. Chen, W. J., L. Gross, K. E. Joho, and W. T. McAllister. 1992. A modified kanamycin-resistance cassette to facilitate twocodon insertion mutagenesis. Gene 111:143-144.

4. Chin, A. M., D. A. Feldheim, and M. H. Saier, Jr. 1989. Altered transcriptional patterns affecting several metabolic pathways in strains of Salmonella typhimurium which overexpress the fructose regulon. J. Bacteriol. 171:2424-2434.

5. Chin, A. M., B. U. Feucht, and M. H. Saier, Jr. 1987. Evidence for the regulation of gluconeogenesis by the fructose phosphotransferase system in Salmonella typhimurium. J. Bacteriol. 169:897-899.

6. Cooper, R. A., and H. L. Kornberg. 1967. The direct synthesis of phosphoenolpyruvate from pyruvate in $E$. coli. Proc. R. Soc. London Ser. B 168:263-280.

7. Cooper, R. A., and H. L. Kornberg. 1969. Phosphoenolpyruvate synthetase. Methods Enzymol. 13:309-314.

8. Cooper, R. A., and H. L. Kornberg. 1974. Phosphoenolpyruvate synthetase and pyruvate phosphate dikinase, p. 631-649. In P. D. Boyer (ed.), The enzymes, 3rd ed., vol. 10. Academic Press, Inc., New York.

9. Czok, R., and W. Lamprecht. 1974. Pyruvate, phosphoenolpyruvate, and D-glycerate-2-phosphate, p. 1446-1451. In H. U. Bergmeyer and K. Gawehn (ed.), Methods of enzymatic analysis, vol. 3. Academic Press, Inc., New York.

10. Daldal, F., and D. G. Fraenkel. 1983. Assessment of a futile cycle involving reconversion of fructose 6-phosphate to fructose 1,6-bisphosphate during gluconeogenic growth of Escherichia coli. J. Bacteriol. 153:390-394.

11. Friedemann, T. E., and G. H. Haugen. 1943. Pyruvic acid. II. The determination of keto acids in blood and urine. J. Biol. Chem. 147:415-442.

12. Geerse, R. H., F. Izzo, and P. W. Postma. 1989. The PEP: fructose phosphotransferase system in Salmonella typhimurium FPr combines enzyme III ${ }^{\text {Fru }}$ and pseudo-HPr activities. Mol. Gen. Genet. 216:517-525.

13. Geerse, R. H., C. R. Ruig, A. R. J. Schuitema, and P. W. Postma. 1986. Relationship between pseudo-HPr and the PEP: fructose phosphotransferase system in Salmonella typhimurium and Escherichia coli. Mol. Gen. Genet. 203:435-444.

14. Geerse, R. H., J. van der Pluijm, and P. W. Postma. 1989. The repressor of the PEP:fructose phosphotransferase system is required for the transcription of the pps gene of Escherichia coli. Mol. Gen. Genet. 218:348-352.

15. Goldie, A. H., and B. D. Sanwal. 1980. Genetic and physiological characterization of Escherichia coli mutants deficient in phosphoenolpyruvate carboxykinase activity. J. Bacteriol. 141: 1115-1121.

16. Groisman, E. A., and M. J. Casadaban. 1986. Mini-Mu bacteriophage with plasmid replicons for in vivo cloning and lac gene fusing. J. Bacteriol. 168:357-364.

17. Ito, K., M. Wittekind, M. Nomura, K. Shiba, T. Yura, A. Miura, and $H$. Nashimoto. 1983. A temperature-sensitive mutant of $E$. coli exhibiting slow processing of exported proteins. Cell 32: 789-797.

18. Kornberg, H. 1986. The roles of HPr and FPr in the utilization of fructose in Escherichia coli. FEBS Lett. 194:12-15.

19. Miller, G. L. 1958. Use of dinitrosalicylic acid reagent for determination of reducing sugars. Anal. Chem. 31:426-428.

20. Miller, J. H. 1972. Experiments in molecular genetics. Cold Spring Harbor Laboratory, Cold Spring Harbor, N.Y.

21. Nakano, S., and T. Onoda. 1989. Effect of protonophore on growth of Escherichia coli. J. Basic Microbiol. 3:163-169.

22. Narindrasorasak, S., and W. A. Bridger. 1977. Phosphoenolpyruvate synthetase of Escherichia coli: molecular weight, subunit composition, and identification of phosphohistidine in phospho-enzyme intermediate. J. Biol. Chem. 252:3121-3127.

23. Niersbach, M., F. Krauzaler, R. H. Geerse, P. W. Postma, and H. J. Hirsch. 1992. Cloning and nucleotide sequence of the Escherichia coli K-12 ppsA gene, encoding PEP synthase. Mol. Gen. Genet. 231:332-336.

24. Saier, M. H., Jr., and A. M. Chin. 1990. Energetics of the bacterial phosphotransferase system in sugar transport and regulation of carbon metabolism, p. 273-294. In T. A. Krulwich (ed.), The bacteria, vol. XII. Bacterial energetics. Academic Press, Inc., New York.

25. Sambrook, J., E. F. Fritsch, and T. Maniatis. 1989. Molecular cloning: a laboratory manual, 2nd ed. Cold Spring Harbor Laboratory, Cold Spring Harbor, N.Y. 